Research Article

\title{
Interface Hepatitis over Grade 2 May Differentiate Chronic Inflammation Associated with CHB from NAFLD in the Early Stage
}

\author{
Yong-fen Zhu, Jin Wang, Jia-zhui Fang, Qiao Yang, and Fang-fang Lv (iD \\ Department of Hepatology and Infection, Sir Run Run Shaw Hospital, Affiliated with School of Medicine, Zhejiang University, \\ Hangzhou 310016, China \\ Correspondence should be addressed to Fang-fang Lv; lvfangfang@zju.edu.cn
}

Received 5 September 2019; Revised 20 February 2020; Accepted 17 March 2020; Published 20 April 2020

Academic Editor: Roberto Caronna

Copyright ( 2020 Yong-fen Zhu et al. This is an open access article distributed under the Creative Commons Attribution License, which permits unrestricted use, distribution, and reproduction in any medium, provided the original work is properly cited.

\begin{abstract}
Background. Patients with chronic hepatitis B (CHB) concomitant with nonalcoholic fatty liver disease (NAFLD) are increasing. Objectives. To identify pathological features that can be used to differentiate between chronic inflammation caused by $\mathrm{CHB}$ and that caused by NAFLD. Methods. Patients with CHB $(n=31)$ needing antiviral treatment, NAFLD $(n=50)$, or CHB-NAFLD $(n=51)$ who underwent biopsy were retrospectively enrolled. Pathological characteristics of chronic inflammation were evaluated using the METAVIR scoring system. The rates of three pathological characteristics were first compared in patients with NAFLD and those with CHB, then compared after fibrosis matching, and were finally compared in CHB-NAFLD patients with different viral loads. Results. The rates of interface hepatitis over grade 2 and fibrosis over grade 2 were significantly higher in the CHB group than in the NAFLD group $(100 \%$ vs. $4 \%$ and $80.6 \%$ vs. $22 \%$; both $P<0.0001)$, while no significant difference was observed in the rate of lobular inflammation over grade 2 between the two groups. After fibrosis matching, in patients with F0-2 fibrosis, the rate of interface hepatitis over grade 2 in CHB was significantly higher than that in NAFLD (100\% vs. $0 \% ; P<0.0001)$. In CHB-NAFLD patients with $\mathrm{F} 0-2$ fibrosis, the rate of interface hepatitis over grade 2 in cases with a high viral load was significantly higher than cases with a low viral load $(66.6 \%$ vs. $0 \% ; P<0.0001)$. The rate of lobular inflammation showed no difference between groups. Conclusion. Interface hepatitis over grade 2 can be used for the differential diagnosis of chronic inflammation associated with CHB or NAFLD in the early stage.
\end{abstract}

\section{Introduction}

Hepatitis B virus (HBV) infection is one of the major causes of chronic liver disease, which ranges from chronic hepatitis to liver cirrhosis and even hepatocellular carcinoma $[1,2]$. It is estimated that HBV infection afflicts approximately 240 million patients with chronic hepatitis $\mathrm{B}(\mathrm{CHB})$ worldwide [3]. CHB is most prevalent in China, which has about 90 million patients with this disease [4]. Nonalcoholic fatty liver disease (NAFLD), another important cause of chronic liver disease, is characterized by fat deposition in hepatocytes ranging from simple steatosis to nonalcoholic steatohepatitis (NASH) and NASH-related cirrhosis [5]. The prevalence of NAFLD ranges from $7.9 \%$ to $54.0 \%$ in Asia, with $20.1 \%$ cases in China [5]. NAFLD is becoming one of the most common liver diseases worldwide $[6,7]$.

With the growing epidemic of NAFLD, an increasing number of patients reportedly have $\mathrm{CHB}$ concomitant with NAFLD [8-10]. The prevalence of NAFLD in CHB is about $13.5-19 \%$ [11]. Chronic inflammation with persistently elevated transaminase levels can occur in both NAFLD and CHB. In CHB patients with NAFLD, chronic hepatitis may be caused by CHB, NAFLD, or both [12,13]. A clinical study of antiviral therapy in CHB patients with NAFLD found that when NAFLD patients were diagnosed with liver biopsy and treated with interferon, NAFLD did not affect the efficacy of antiviral therapy, whereas when NAFLD patients were diagnosed with Doppler ultrasound and treated with nucleoside 
analogues, NAFLD affected the efficacy of antiviral therapy, resulting in poor biochemical and virological response [1]. What causes the difference in the treatment outcome, the antiviral treatment regimen (interferon $v s$. nucleoside analogues), or the different methods assessing chronic inflammation (liver biopsy vs. alanine aminotransferase (ALT) and Doppler ultrasound) remains unclear. Doppler ultrasound and abnormal ALT cannot be used for the assessment of the etiology of chronic inflammation. Some researchers have speculated that the differences may be due to NAFLDinduced elevated transaminase being misdiagnosed as $\mathrm{CHB}$ for antiviral therapy [14].

CHB is pathologically characterized by inflammation in the portal area and the surrounding area. Determining whether or not patients should receive antiviral treatment is based on the severity of inflammation and fibrosis, which are graded using the METAVIR scoring system $[15,16]$. The METAVIR score composes of the degree of interface hepatitis, lobular inflammation, and portal fibrosis and can be applied for the grading of inflammation and fibrosis for deciding further antiviral treatment. NAFLD is pathologically characterized by lobular inflammation and balloon-like degeneration, which is assessed using the NAFLD activity score (NAS) [17]. When CHB combined with NAFLD occurs, chronic inflammation caused by CHB is characterized by more severe inflammation in the portal area [11]. However, there have been no reports about the detailed pathological differences between CHB with and without NAFLD.

Generally, patients with chronic inflammation caused by NAFLD are subjected to lifestyle intervention, whereas patients with $\mathrm{CHB}$ are subjected to anti-HBV treatment $[18,19]$. Therefore, when treating chronic inflammation in patients with $\mathrm{CHB}$ complicated with NAFLD, the etiology of chronic inflammation is important for determining the appropriate treatment, especially antiviral therapy for $\mathrm{CHB}$, because inappropriate anti-HBV treatment results in the economic burden of long-term medication and long-term drug safety.

This study analyzed the pathological features of chronic inflammation of NAFLD and CHB, which are needed for antiviral treatment, to identify the pathological features associated with chronic inflammation and to differentiate $\mathrm{CHB}$ from NAFLD. We also confirmed the pathological features in patients with $\mathrm{CHB}$ combined with NAFLD and different viral loads.

\section{Materials and Methods}

2.1. Patients. Patients who underwent liver biopsy for liver pathology between January 2017 and December 2019 in Sir Run Run Shaw Hospital (Zhejiang, China) were enrolled. Patients with $\mathrm{CHB}$ requiring antiviral treatment, NAFLD, or CHB combined with NAFLD were included. Patients with NAFLD were diagnosed as follows [19-21]: had persistent liver function abnormalities for more than 6 months; ultrasound showing fatty liver; liver biopsy showing hepatic steatosis $>5 \%$; and no history of drinking, medication, and viral hepatitis. Patients with $\mathrm{CHB}$ were diagnosed as follows $[22,23]$ : hepatitis B surface antigen- (HBsAg-) positive for
6 months or more; hepatitis $\mathrm{B}$ e antigen- (HBeAg-) positive and HBVDNA > $20000 \mathrm{IU} / \mathrm{mL}$ or HBeAg-negative and HBVDNA > $2000 \mathrm{IU} / \mathrm{mL}$; ultrasound showing no fatty liver; liver biopsy showing hepatic steatosis $>5 \%$; needed antiviral treatment according to METAVIR scores; and antiviral treatment naïve. Patients with $\mathrm{CHB}$ combined with NAFLD were diagnosed as follows: repeated abnormal liver function; HBsAg-positive for 6 months; liver biopsy showing hepatic steatosis $>5 \%$; and no alcoholic liver disease or immune liver disease. Information including gender, age, body mass index (BMI), alanine aminotransferase (ALT), aspartate aminotransferase (AST), rate of HBeAg positivity, hypertension, diabetes, and hyperlipidemia was retrospectively collected from the electronic case records. The study protocol was approved by the Ethics Committee of Sir Run Run Shaw Hospital. Each participant provided written informed consent.

\subsection{Liver Biopsy and Histopathology Assessment. Liver tissue} with a length of about $1.0-2.0 \mathrm{~cm}$ was obtained using a $16 \mathrm{G}$ puncture needle through vacuum suction under ultrasonic localization. The tissue was fixed in $4 \%$ dehydrated, embedded in paraffin, and sectioned continuously. The slides were subjected to hematoxylin and eosin (H\&E) staining and silver staining of the reticular fiber and then read by an experienced pathologist. The METAVIR scoring system is the standard semiquantitative pathology criteria for whether antiviral treatment is needed for CHB patients. It includes the scores of interface hepatitis, lobular inflammation, and portal fibrosis. Therefore, in the present study, interface hepatitis, lobular inflammation, and portal fibrosis were assessed using the METAVIR scoring system [15], and steatosis was evaluated using the NAS scoring system [17]. Liver histology was defined as follows. Interface hepatitis (also known as piecemeal necrosis) was divided into four grades (0-3): 0 , absent; 1, local inflammation of the periportal plate in some portal tract; 2 ,diffuse alteration of the periportal plate in some portal tract or focal lesions around all portal areas; and 3, diffuse alteration of the periportal plate in all portal areas. Lobular inflammation was divided into three grades (0-2): 0 , one focus or less per hepatic lobule; 1 , at least 1 focus per hepatic lobule; and 2, several foci per hepatic lobule or bridging necrosis and fusion necrosis. Fibrosis was divided into five grades (F0-4): 0 , absent; 1 , portal fibrosis without septa; 2, portal fibrosis with rare septa; 3 , numerous septa without cirrhosis; and 4 , cirrhosis. Steatosis was divided into four grades (S0-3): $0,<5 \% ; 1,5-33 \%$ (including 33); , $33-66 \%$; and $3,>66 \%$.

2.3. Statistical Analysis. Results are presented as medians (interquartile range) for continuous variables and percentage values for categorical variables. Statistical analysis was performed using SPSS 17.0 for Windows (SPSS, Inc., Chicago, IL, USA). $P$ values were determined by Fisher's exact test for categorical variables and Wilcoxon rank-sum test for continuous variables. $P<0.05$ was considered statistically significant. 
TABLE 1: Baseline characteristics of the participants.

\begin{tabular}{lccccc}
\hline & & & & \multicolumn{2}{c}{ value } \\
& NAFLD $(n=50)$ & CHB $(n=31)$ & CHB and NAFLD $(n=51)$ & NAFLD vs. CHB & NAFLD vs. CHB and NAFLD \\
\hline Gender (female/male) & $13 / 37$ & $8 / 23$ & $8 / 43$ & 0.985 & 0.202 \\
Age & $37.72 \pm 10.49$ & $38.90 \pm 7.49$ & $41.29 \pm 10.46$ & 0.363 & 0.115 \\
BMI & $27.99 \pm 3.19$ & $21.58 \pm 2.38$ & $25.62 \pm 3.45$ & $<0.0001$ & $<0.0001$ \\
ALT & $117.78 \pm 84.17$ & $68.35 \pm 68.01$ & $62.25 \pm 50.80$ & $<0.0001$ & $<0.0001$ \\
AST & $56.20 \pm 31.81$ & $42.32 \pm 36.26$ & $35.80 \pm 20.15$ & 0.001 & $<0.0001$ \\
HBeAg-positive rate & $/$ & $15 / 31$ & $19 / 51$ & 0.321 & $/$ \\
Hypertension & $7 / 50$ & $/$ & $9 / 51$ & $/$ & 0.616 \\
Diabetes & $6 / 50$ & $/$ & $6 / 51$ & $/$ & 0.971 \\
Hyperlipidemia & $46 / 50$ & $/$ & $24 / 51$ & $/$ & $<0.0001$ \\
Steatosis & $17 / 50$ & $/$ & $37 / 51$ & & $<0.0001$ \\
S1 & $27 / 50$ & $/$ & $13 / 51$ & $1 / 51$ & \\
S2 & $6 / 50$ & $/$ & & & \\
S3 & & & &
\end{tabular}

NAFLD: nonalcoholic fatty liver disease; CHB: chronic hepatitis B; BMI: body mass index; ALT: alanine aminotransferase; AST: aspartate aminotransferase; HBeAg: HBV e antigen.

\section{Results}

3.1. Baseline Characteristics of the Participants. A total of 132 participants were included, with 50, 31, and 51 cases of NAFLD, CHB, and CHB combined with NAFLD, respectively. The baseline characteristics of the patients are shown in Table 1. Significant differences were observed among the three groups in BMI $(27.99 \pm 3.19$ vs. $21.58 \pm 2.38$ vs. 25.62 $\pm 3.45, P<0.0001$ ) and transaminase levels (ALT $117.78 \pm$ 84.17 vs. $68.35 \pm 68.01$ vs. $62.25 \pm 50.80, P<0.0001$; AST $56.20 \pm 31.81$ vs. $42.32 \pm 36.26$ vs. $35.80 \pm 20.15, P=0.001)$. The rate of hyperlipidemia in the NAFLD group was higher than that in the $\mathrm{CHB}$ combined with the NAFLD group (46/50 vs. 24/51, $P<0.0001)$. Similarly, hepatic steatosis was more severe in the NAFLD group.

3.2. Comparison of Pathological Features between $\mathrm{CHB}$ and NAFLD. As shown in Table 2, the rate of interface hepatitis over grade 2 was significantly higher in the $\mathrm{CHB}$ group than in the NAFLD group $(100 \%(31 / 31)$ vs. $4 \%(2 / 50) ; P<0.0001)$. The rate of portal fibrosis over grade 2 was also significantly higher in the CHB group than in the NAFLD group $(80.6 \%$ $(25 / 31)$ in the CHB group vs. $22 \%(11 / 50)$ in the NAFLD group). No significant difference was observed in the rate of lobular inflammation over grade 2 between the two groups (41.9\% (13/31) vs. $24 \%(12 / 50) ; P=0.091)$.

3.3. Comparison of Pathological Features between $\mathrm{CHB}$ and NAFLD after Fibrosis Hierarchical Matching. There were two cases of interface hepatitis over grade 2 in the NAFLD group, and all cases presented with fibrosis over F2. The rates of interface hepatitis and lobular inflammation were compared between the NAFLD and CHB groups in patients with fibrosis less than F2 after fibrosis matching. As shown in Table 3, none of the 45 patients with NAFLD showed interface hepatitis over grade 2, whereas all 23 patients with $\mathrm{CHB}$ showed interface hepatitis over grade $2(P<0.0001)$.
TABLE 2: Inflammatory histopathology of CHB and NAFLD.

\begin{tabular}{lccc}
\hline & NAFLD $(n=50)$ & CHB $(n=31)$ & $P$ value \\
\hline Interface hepatitis & & & $<0.0001$ \\
Grade 0 & $46(92 \%)$ & 0 & \\
Grade 1 & $2(4 \%)$ & 0 & \\
Grade 2 & $2(4 \%)$ & $27(87.1 \%)$ & \\
Grade 3 & 0 & $4(12.9 \%)$ & \\
Lobular inflammation & & & 0.091 \\
Grade (0, 1) & $38(76 \%)$ & $18(58.1 \%)$ & \\
Grade (2) & $12(24 \%)$ & $13(41.9 \%$ & \\
Fibrosis & & & $<0.0001$ \\
Grade 0 & $22(44 \%)$ & 0 & \\
Grade 1 & $17(34 \%)$ & $6(19.4 \%)$ & \\
Grade 2 & $6(12 \%)$ & $17(54.8 \%)$ & \\
Grade 3 & $5(10 \%)$ & $7(22.6 \%)$ & \\
Grade 4 & 0 & $1(3.2 \%)$ & \\
\hline
\end{tabular}

No significant difference was observed in the rate of lobular inflammation between groups.

3.4. Pathological Characteristics of Patients with CHB Combined with NAFLD. Patients were divided into two groups according to HBV DNA load, low viral load group (HBVDNA $<2000 \mathrm{IU} / \mathrm{mL}, n=19)$ and high viral load group (HBVDNA $\geq 2000 \mathrm{IU} / \mathrm{mL}, n=21$ ). As shown in Table 4 , the rates of interface hepatitis and lobular inflammation were compared between both groups in patients with fibrosis less than F2. The rate of interface hepatitis over grade 2 was $66.6 \%$ in the high viral load group, whereas there was no interface hepatitis in the low viral load group $(P<0.0001)$. The rate of lobular inflammation showed no difference between groups. Liver pathology pictures of interface hepatitis in each group are shown in Figure 1. 
TABLE 3: Inflammatory histopathology of CHB and NAFLD after fibrosis hierarchical matching.

\begin{tabular}{lccc}
\hline & $\begin{array}{c}1 \\
\text { NAFLD with severe fibrosis } \\
(\mathrm{F} 3-4, n=5)\end{array}$ & $\begin{array}{c}2 \\
\text { NAFLD with mild-moderate fibrosis } \\
(\mathrm{F} 0-2, n=45)\end{array}$ & $\begin{array}{c}\text { CHB with mild-moderate fibrosis } \\
(\mathrm{F} 0-2, N=23)\end{array}$ \\
$\begin{array}{l}\text { Interface hepatitis } \\
\text { Grade 0 }\end{array}$ & $3(60 \%)$ & $43(95.6 \%)$ & 0 \\
Grade 1 & 0 & $2(3.7 \%)$ & 0 \\
Grade 2 & $2(40 \%)$ & 0 & $21(91.3 \%)$ \\
Grade 3 & 0 & 0 & $2(8.7 \%)$ \\
Lobular inflammation & $3(60 \%)$ & $33(73.3 \%)$ & $14(60.9 \%)$ \\
Grade $(0,1)$ & $2(40 \%)$ & $12(26.7 \%)$ & $9(39.1 \%)$ \\
Grade 2 & & & 0.296
\end{tabular}

TABLE 4: Inflammatory histopathology of CHB combined with NAFLD CHB in patients with different vial loads.

\begin{tabular}{lcc}
\hline & $\begin{array}{c}\text { HBV DNA }>\text { E4 IU/mL, mild-moderate } \\
\text { fibrosis F0-2 }(n=21)\end{array}$ & $\begin{array}{c}\text { HBV DNA < E4 IU/mL, mild-moderate } \\
\text { fibrosis F0-2 }(n=19)\end{array}$ \\
\hline Interface hepatitis & $3(14.3 \%)$ & $16(84.2 \%)$ \\
Grade 0 & $4(19 \%)$ & $3(15.8 \%)$ \\
Grade 1 & $12(57.1 \%)$ & 0 \\
Grade 2 & $2(9.5 \%)$ & 0 \\
Grade 3 & & $15(78.9 \%)$ \\
Lobular inflammation & $13(61.9 \%)$ & $4(21.1 \%)$ \\
Grade $(0,1)$ & $8(38.1 \%)$ & 0.0001 \\
Grade 2 &
\end{tabular}

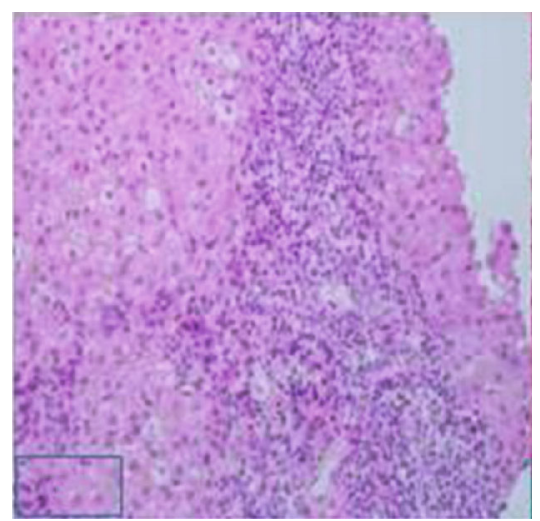

(a)

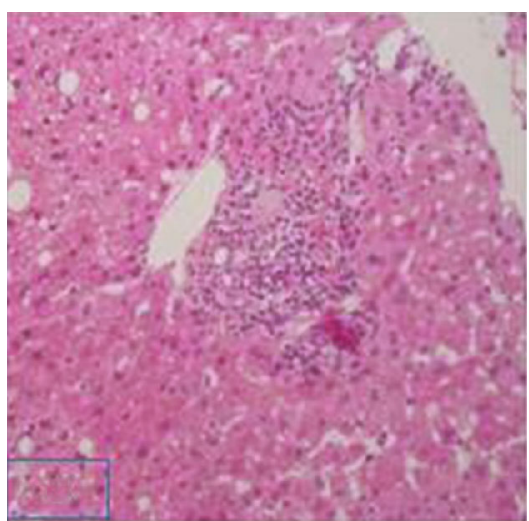

(b)

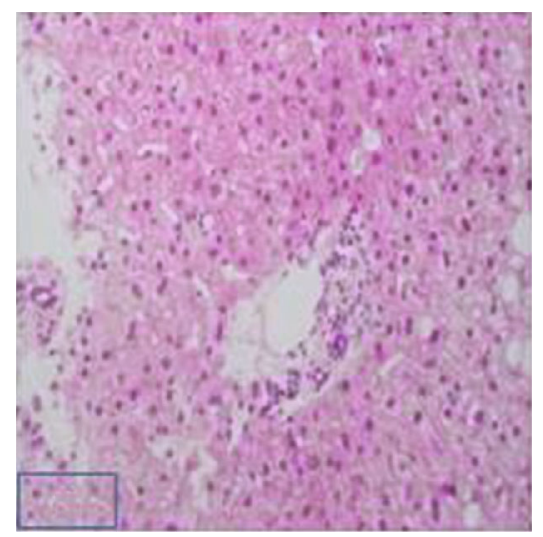

(c)

FIGURE 1: Representative pictures of interfacial hepatitis. (a) Male, 27 years old, chronic HBV infection, ALT 66 U/L, HBV DNA $1.58 *$ E8 $\mathrm{IU} / \mathrm{mL}$; liver biopsy revealed grade 3 interface hepatitis, grade 2 fibrosis, and hepatic steatosis 40-50\% (S2), being diagnosed with CHB combined with NAFLD; (b) male, 34 years old, chronic HBV infection, ALT 47 IU/L, HBV DNA E7 IU/mL, liver biopsy revealed grade 1 interface hepatitis, grade 1-2 fibrosis, hepatic steatosis 50-60\% (S2), being diagnosed with NAFLD while infected with HBV; (c), Male, 36 years old, chronic HBV infection, HBVDNA 57, ALT $58 \mathrm{U} / \mathrm{L}$; liver biopsy revealed grade 0 interface hepatitis, grade 0 fibrosis, and hepatic steatosis $30 \%$ (S1), being diagnosed with NAFLD while infected with HBV.

\section{Discussion}

In the present study, the histopathology features including interface hepatitis, lobular inflammation, and fibrosis were compared between patients with $\mathrm{CHB}$ and NAFLD. The features were further compared after fibrosis matching. We found that patients with $\mathrm{CHB}$ showed a higher rate of interface hepatitis over grade 2. Further study in patients 
with CHB combined with NAFLD showed that the rate of interface hepatitis over grade 2 was higher in the high viral load group than in the low viral load group.

Inflammation in the portal area includes inflammatory cell infiltration in the portal area and piecemeal necrosis (interface hepatitis) caused by infiltration of inflammatory cells into the liver cells. The pathological characteristics of $\mathrm{CHB}$ are the portal and surrounding inflammation. For patients with $\mathrm{CHB}$, interface hepatitis, lobular inflammation, and portal fibrosis should be evaluated according to the METAVIR scoring system, and patients with inflammation over grade 2 as well as fibrosis over grade 2 should be subjected to antiviral therapy $[15,16,18]$. Inflammation in the portal area has not been evaluated in adult NAFLD. However, lymphocytic infiltration in the portal area is considered to be the pathological feature of progressive NAFLD [24]. Studies about interface hepatitis in NASH are not available yet.

For CHB patients, inflammation over grade 2 and portal fibrosis over grade 2 indicate that the patient has entered the reactivation phase during HBV infection and anti-HBV therapy is required $[8,11]$. Thus, the rates of interface hepatitis, lobular inflammation, and fibrosis over grade 2 were compared in the present study. Our data showed that the rates of interface hepatitis over grade 2 and fibrosis over grade 2 were significantly higher in the $\mathrm{CHB}$ group than in the NAFLD group, while no significant difference was observed in the rate of lobular inflammation over grade 2 between the two groups. These results suggest that lesions in the portal area of the liver of CHB patients were significantly more severe than those in NAFLD patients, which is consistent with a previous report about the pathological features of CHB and NAFLD [17].

In the NAFLD group, two patients with interface hepatitis over grade 2 both showed significant hepatic fibrosis. A previous study showed that lymphocytic infiltration in the portal area in patients with NAFLD suggests the risk of disease progression [24]. We also observed more severe portal inflammation-interface hepatitis in patients with cirrhosis or precirrhosis, further confirming the above hypothesis that inflammation in the portal area indicates the progression of NAFLD. None of the 45 cases of NAFLD with mild to middle fibrosis (F0-2) showed interface hepatitis over grade 2, whereas all paired 23 cases of $\mathrm{CHB}$ with mild to middle fibrosis (F0-2) showed interface hepatitis over grade 2. However, the rate of lobular inflammation was not significantly different between the two groups. Therefore, this study suggests that for patients with early-stage chronic hepatitis (fibrosis $\leq$ grade 2), interface hepatitis over grade 2 can be used as the pathological feature of diagnosis for differentiating patients with $\mathrm{CHB}$ in the immune clearance phase from patients with NAFLD. Interface hepatitis is the typical pathological features of $\mathrm{CHB}$ and is the liver damage caused by the activation of the acquired immune T lymphocytes. However, NAFLD is the inflammation caused by the macrophage activation of the natural immune system. The main pathological features of NAFLD are the lobular inflammation and ballooning degeneration of hepatocyte, and the activation of the acquired immune system may also be involved later. Therefore, we speculated that in NAFLD, the activation of acquired immune system and the occurrence of the interface hepatitis and bile duct reaction are intermediate processes promoting liver fibrosis, finally leading to liver cirrhosis [25-27].

The differential diagnostic ability of interface hepatitis over grade $2 \mathrm{CHB}$ and NAFLD was further validated in patients with $\mathrm{CHB}$ concomitant with NAFLD. Patients with HBVDNA $<2000 \mathrm{IU} / \mathrm{mL}$ reportedly have a low risk of CHB [28]. Thus, these patients with persistent abnormal liver function were divided into high viral load and low viral load groups using $2000 \mathrm{IU} / \mathrm{mL}$ as the cutoff value. None of the 19 patients with a low viral load showed interface hepatitis grade 2 , suggesting that chronic inflammation in these patients may be caused by NAFLD, which is consistent with a previous study showing that chronic inflammation in patients with CHB and NAFLD coexistent with low viral load may be due to NAFLD [13]. The rate of interface hepatitis over grade 2 in patients with a high viral load was $66.6 \%$, suggesting that chronic inflammation in these patients was mainly because of $\mathrm{CHB}$, and chronic inflammation in the remaining $33.3 \%$ of these patients may be caused by NAFLD, and no antiviral treatment is needed. If a liver biopsy is not performed and antiviral treatment is only based on a transaminase abnormality, the $33.3 \%$ of patients may be treated using the wrong regimen. Our results explained that if only a transaminase abnormality is used as the indication for antiviral treatment in CHB and NAFLD coexistent patients, some chronic inflammation caused by NAFLD may be wrongly treated by antiviral therapy, resulting in poor response [1].

In the present study, baseline transaminase levels were significantly different among three patient groups. The rates of steatosis in the different grades were also different. These differences were due to the different reasons for biopsy in the different patient groups. CHB patients who were enrolled in this study underwent biopsy because the levels of transaminase were not high enough (twice the upper limit of normal) to receive antiviral treatment, and antiviral treatment was performed after diagnosis using liver pathology. Thus, the transaminase level was low. Patients in the NAFLD group and the CHB combined with the NAFLD group underwent biopsy because of long-term transaminase abnormalities without a clear cause. However, liver pathology was used as the gold standard here, so the difference in baseline transaminase should not affect the results.

There were several limitations in this study. First, this was a single-center study with a small number of patients enrolled. A multicenter study with more patients is needed to further validate the results. Second, this was a crosssectional study. A cohort study with treatment and followup is needed to validate its clinical significance for differential diagnosis.

\section{Conclusions}

In conclusion, by comparing the pathological characteristics of CHB and NAFLD, we found that in chronic liver inflammation at the early stage (F0-2), interface hepatitis over 
grade 2 can be used as a differential diagnosis feature for $\mathrm{CHB}$ and NAFLD, which may provide guidance when treating patients with $\mathrm{CHB}$ concomitant NAFLD in the early stage of inflammation.

\section{Abbreviations}

HBV: Hepatitis B virus

CHB: Chronic hepatitis B

NAFLD: Nonalcoholic fatty liver disease

NASH: Nonalcoholic steatohepatitis

NAS: NAFLD activity score.

\section{Data Availability}

The data sets generated and analyzed during the present study are available from the corresponding author on reasonable request.

\section{Ethical Approval}

The study protocol was approved by the Ethics Committee of Sir Run Run Shaw Hospital.

\section{Consent}

All data published here are under the consent for publication.

\section{Conflicts of Interest}

We declare that we have no conflict of interest.

\section{Authors' Contributions}

Yong-fen Zhu and Fang-fang Lv designed most of the investigation, analyzed data, and wrote the manuscript; Jin Wang provided pathological assistance; Jia-zhui Fang and Qiao Yang contributed to the interpretation of the data and analyses. All of the authors have read and approved the manuscript.

\section{Acknowledgments}

This work was supported by Zhejiang Provincial Natural Science Foundation of China (no. LY15H030009).

\section{References}

[1] Y. Zhu, Q. Yang, F. Lv, and Y. Yu, "The effect of hepatosteatosis on response to antiviral treatment in patients with chronic hepatitis B: a meta-analysis," Gastroenterology Research and Practice, vol. 2017, Article ID 1096406, 12 pages, 2017.

[2] C. Seeger and W. S. Mason, "Molecular biology of hepatitis B virus infection,” Virology, vol. 479-480, pp. 672-686, 2015.

[3] R. G. Gish, B. D. Given, C. L. Lai et al., "Chronic hepatitis B: virology, natural history, current management and a glimpse at future opportunities," Antiviral Research, vol. 121, pp. 47-58, 2015.

[4] H. Ren, "The experience of management of chronic hepatitis B in China," Journal of Viral Hepatitis, vol. 24, Suppl 1, pp. 4-5, 2017.
[5] W. K. Seto and M. F. Yuen, "Nonalcoholic fatty liver disease in Asia: emerging perspectives," Journal of Gastroenterology, vol. 52, no. 2, pp. 164-174, 2017.

[6] R. Loomba and A. J. Sanyal, "The global NAFLD epidemic," Nature Reviews. Gastroenterology \& Hepatology, vol. 10, no. 11, pp. 686-690, 2013.

[7] Z. Younossi, Q. M. Anstee, M. Marietti et al., "Global burden of NAFLD and NASH: trends, predictions, risk factors and prevention," Nature Reviews. Gastroenterology \& Hepatology, vol. 15, no. 1, pp. 11-20, 2018.

[8] R. Pais, E. Rusu, D. Zilisteanu et al., "Prevalence of steatosis and insulin resistance in patients with chronic hepatitis B compared with chronic hepatitis $\mathrm{C}$ and non-alcoholic fatty liver disease," European Journal of Internal Medicine, vol. 26, no. 1, pp. 30-36, 2015.

[9] M. M. Wang, G. S. Wang, F. Shen, G. Y. Chen, Q. Pan, and J. G. Fan, "Hepatic steatosis is highly prevalent in hepatitis B patients and negatively associated with virological factors," Digestive Diseases and Sciences, vol. 59, no. 10, pp. 25712579, 2014.

[10] V. W. Wong, G. L. Wong, W. C. Chu et al., "Hepatitis B virus infection and fatty liver in the general population," Journal of Hepatology, vol. 56, no. 3, pp. 533-540, 2012.

[11] R. Kumar and G. Boon-Bee Goh, "Chronic hepatitis B and fatty liver: issues in clinical management," Clinics and Research in Hepatology and Gastroenterology, vol. 40, no. 6, pp. 755759, 2016.

[12] P. R. Spradling, L. Bulkow, E. H. Teshale et al., "Prevalence and causes of elevated serum aminotransferase levels in a population-based cohort of persons with chronic hepatitis B virus infection," Journal of Hepatology, vol. 61, no. 4, pp. 785-791, 2014.

[13] K. Demir, F. Akyuz, S. Ozdil et al., "What is the reason of elevated alanine aminotransferase level in $\mathrm{HBeAg}$ negative patients with low viremia: NAFLD or chronic hepatitis?," Annals of Hepatology, vol. 6, no. 2, pp. 92-96, 2007.

[14] X. Jin, Y. P. Chen, Y. D. Yang, Y. M. Li, L. Zheng, and C. Q. Xu, "Association between hepatic steatosis and entecavir treatment failure in Chinese patients with chronic hepatitis B," PLoS One, vol. 7, no. 3, article e34198, 2012.

[15] P. Bedossa and T. Poynard, "An algorithm for the grading of activity in chronic hepatitis C. The METAVIR Cooperative Study Group," Hepatology, vol. 24, no. 2, pp. 289-293, 1996.

[16] K. G. Ishak, "Pathologic features of chronic hepatitis. A review and update," American Journal of Clinical Pathology, vol. 113, no. 1, pp. 40-55, 2000.

[17] D. E. Kleiner and H. R. Makhlouf, "Histology of nonalcoholic fatty liver disease and nonalcoholic steatohepatitis in adults and children," Clinics in Liver Disease, vol. 20, no. 2, pp. 293-312, 2016.

[18] European Association for the Study of the Liver, "EASL 2017 clinical practice guidelines on the management of hepatitis B virus infection," Journal of Hepatology, vol. 67, pp. 370-398, 2017.

[19] N. Chalasani, Z. Younossi, J. E. Lavine et al., "The diagnosis and management of nonalcoholic fatty liver disease: practice guidance from the American Association for the Study of Liver Diseases," Hepatology, vol. 67, no. 1, pp. 328-357, 2018.

[20] F. Jian-gao and Chinese Liver Disease Association, "Guidelines for management of nonalcoholic fatty liver disease: an updated 
and revised edition," Zhonghua Gan Zang Bing Za Zhi, vol. 18, no. 3, pp. 163-166, 2010.

[21] N. Chalasani, Z. Younossi, J. E. Lavine et al., "The diagnosis and management of non-alcoholic fatty liver disease: practice guideline by the American Association for the Study of Liver Diseases, American College of Gastroenterology, and the American Gastroenterological Association," Hepatology, vol. 55, no. 6, pp. 2005-2023, 2012.

[22] N. A. Terrault, A. S. F. Lok, B. J. McMahon et al., "Update on prevention, diagnosis, and treatment of chronic hepatitis $\mathrm{B}$ : AASLD 2018 hepatitis B guidance," Hepatology, vol. 67, no. 4, pp. 1560-1599, 2018.

[23] Chinese Society of Hepatology CMA, Chinese Society of Infectious Diseases CMA, J. L. Hou, and W. Lai, "The guideline of prevention and treatment for chronic hepatitis B: a 2015 update," Zhonghua Gan Zang Bing Za Zhi, vol. 23, pp. 888905, 2015.

[24] E. M. Brunt, D. E. Kleiner, L. A. Wilson et al., "Portal chronic inflammation in nonalcoholic fatty liver disease (NAFLD): a histologic marker of advanced NAFLD-clinicopathologic correlations from the nonalcoholic steatohepatitis clinical research network," Hepatology, vol. 49, no. 3, pp. 809-820, 2009.

[25] J. Y. N. Lau, X. Xie, M. M. C. Lai, and P. C. Wu, “Apoptosis and viral hepatitis," Seminars in Liver Disease, vol. 18, no. 2, pp. 169-176, 1998.

[26] S. Sutti, S. Bruzzì, and E. Albano, "The role of immune mechanisms in alcoholic and nonalcoholic steatohepatitis: a 2015 update," Expert Review of Gastroenterology \& Hepatology, vol. 10, no. 2, pp. 243-253, 2016.

[27] V. L. Gadd, R. Skoien, E. E. Powell et al., "The portal inflammatory infiltrate and ductular reaction in human nonalcoholic fatty liver disease," Hepatology, vol. 59, no. 4, pp. 1393-1405, 2014.

[28] G. V. Papatheodoridis, S. Manolakopoulos, Y. F. Liaw, and A. Lok, "Follow-up and indications for liver biopsy in HBeAg-negative chronic hepatitis B virus infection with persistently normal ALT: a systematic review," Journal of Hepatology, vol. 57, no. 1, pp. 196-202, 2012. 